\title{
Gelfand duality for compact pospaces
}

\author{
Laurent De Rudder and Georges Hansoul \\ Departement of Mathematics, University of Liege \\ 1.derudder@ulg.ac.be
}

Let $X$ be a compact Hausdorff space. It is well known that $X$ can be characterized by its ring of real continuous functions, by its set of regular open subsets or more simply by its set of open subsets. These characterizations lead to dualities between the category KHaus, of compact Hausdorff space and respectively the categories $\mathbf{C}^{\star}$-alg (or equivalently ubal), of commutative $C^{\star}$-algebras, DeV of de Vries algebras and KRFrm of compact regular frames. We thus get a square of dualities. (see [1], [2] and [6]).

Later, G.Bezhanishvili and J.Harding extended in [1] a part square to dualities between the categories StKSp of stably compact spaces, RPrFrm of regular proximity frames and StKFrm of stably compact frames.

We thus get the square of dualities extended this way.



Our aim is to complete the outside triangle, looking for a category generalizing the $C^{\star}$ algebras.

Using the equivalences between StKSp and the category KPSp of compact po-spaces (see [4]), an essential fact, due to G.Hansoul in [5] leads us to consider a category of ordered semiring. Indeed, we can see that the Nachbin-Stone-Cech compactification of a completely regular ordered po-space $X$ can be realized through its semi-ring of increasing, continuous and real, positive functions, denoted $I\left(X, \mathbb{R}^{+}\right)$.

Following the definitions of G.Bezhanishvili, P.Morandi and B.Olberding in [2], we define the bounded Archimedean $\ell$-semi-algebras this way.

Definition 1. 1. An $\ell$-semi-ring is an algebra $(A,+, ., 0,1, \leq)$ with the following axioms :

(a) $(A,+, 0)$ and $(A, ., 1)$ are commutative monoids.

(b) $(A,+,$.$) is distributive.$

(c) $a \leq b \Leftrightarrow a+c \leq b+c$.

(d) $a \geq 0$ and $a \leq b \Rightarrow a . c \leq b . c$

(e) $(A, \leq)$ is a lattice.

2. An $\ell$-semi-ring $A$ is bounded if for all $a \in A$, there is $n \in \mathbb{N}$ such that $a \leq n .1$.

3. An $\ell$-semi-ring $A$ is Archimedean if for all $a, b, c, d \in A$, whenever $n . a+b \leq n . c+d$, then $a \leq c$. 
4. An $\ell$-semi-ring $A$ is an $\ell$-semi-algebra if it is an $\mathbb{R}^{+}$-algebra such that for all $a, b \in A$ and $r \in \mathbb{R}^{+}, r . a \leq r . b$.

5. $(a \vee b)+c=(a+c) \vee(b+c)$ and $(a \wedge b)+c=(a+c) \wedge(b+c)$.

We now denote sbal the category of bounded Archimedean $\ell$-semi-algebras, and defining the morphisms in the natural way.

In order to get the missing duality, we define the $\sim$-relation on $A \times A$, with $A$ an sbal, such as

$$
(a, b) \sim(c, d) \Leftrightarrow a+d=b+c,
$$

allowing us to construct the functor ${ }^{b}:$ sbal $\longrightarrow$ bal which sends $A$ to $A \times A / \sim$. In particular, this functor enable us to easily transfer structures from rings to semi-rings.

With all these tools, we propose the following functors between KPSp and sbal : the first functor, denoted $I$, sends a compact po-space $X$ to the set $I\left(X, \mathbb{R}^{+}\right)$and a continuous increasing function $f: X \longrightarrow Y$ between compact po-spaces to

$$
f_{\star}: I\left(Y, \mathbb{R}^{+}\right) \longrightarrow I\left(X, \mathbb{R}^{+}\right): g \longmapsto g \circ f .
$$

On the other side, the second functor, denoted $\chi$, maps a sbal $A$ to its set of $\ell$-congruences, denoted $X_{A}$ and a morphism $\alpha: A \longrightarrow B$ between sbals to

$$
\alpha^{\star}: X_{B} \longrightarrow X_{A}
$$

such that, if $\theta \in X_{B},(a, b) \in \alpha^{\star}(\theta)$ if and only if $(\alpha(a), \alpha(b)) \in \theta$.

Definition 2. An $\ell$-semi-ring $A$ admits difference with constants if for all $a \in A$ and $r \in \mathbb{R}^{+}$ $a \leq r .1$ implies there is $b \in A$ such that $a=b+r .1$. It is uniformly complete if it is complete for the norm $\|a\|=\inf \{\lambda \in \mathbb{R}: a \leq \lambda .1\}$. We then denote usbal the full subcategory of sbal whose objects are the uniformly complete bounded Archimedean $\ell$-semi-algebras with difference with constants.

Theorem 3. The functors $\chi$ and I establish a dual equivalence between usbal and KPSp

\section{References}

[1] Guram Bezhanishvili and John Harding. Proximity frames and regularization. Appl. Categ. Struct., $22(1): 43-78,2014$.

[2] Guram Bezhanishvili, Patrick J. Morandi, and Bruce Olberding. Bounded Archimedean $\ell$-algebras and Gelfand-Neumark-Stone duality. Theory Appl. Categ., 28:435-475, 2013.

[3] Israel Gelfand and Mark Neumark. On the imbedding of normed rings into the ring of operators in Hilbert space. Mat. Sb., Nov. Ser., 12:197-213, 1943.

[4] Gerhard Gierz, Karl Hofmann, Klaus Keimel, Jimmie Lawson, Michael Mislove, and Dana S. Scott. Continuous lattices and domains. Cambridge: Cambridge University Press, 2003.

[5] Georges Hansoul. The Stone-Čech compactification of a pospace. Universal algebra, Colloq., Szeged/Hung. 1983, Colloq. Math. Soc. János Bolyai 43, 161-176 (1986)., 1986.

[6] Peter T. Johnstone. Stone spaces, volume 3 of Cambridge Studies in Advanced Mathematics. Cambridge University Press, 1982.

[7] Marshall H. Stone. A general theory of spectra. I. Proc. Natl. Acad. Sci. USA, 26:280-283, 1940. 